\title{
Amyloid Imaging: Poised for Integration into Medical Practice
}

\author{
Keshav Anand $^{1} \cdot$ Marwan Sabbagh $^{2}$
}

Published online: 29 August 2016

(C) The American Society for Experimental NeuroTherapeutics, Inc. 2016

\begin{abstract}
Amyloid imaging represents a significant advance as an adjunct in the diagnosis of Alzheimer's disease (AD) because it is the first imaging modality that identifies in vivo changes known to be associated with the pathogenesis. Initially, ${ }^{11} \mathrm{C}-\mathrm{PIB}$ was developed, which was the prototype for many ${ }^{18} \mathrm{~F}$ compounds, including florbetapir, florbetaben, and flutemetamol, among others. Despite the high sensitivity and specificity of amyloid imaging, it is not commonly used in clinical practice, mainly because it is not reimbursed under current Center for Medicare and Medicaid Services guidelines in the USA. To guide the field in who would be most appropriate for the utility of amyloid positron emission tomography, current studies are underway [Imaging Dementia Evidence for Amyloid Scanning (IDEAS) Study] that will inform the field on the utilization of amyloid positron emission tomography in clinical practice. With the advent of monoclonal antibodies that specifically target amyloid antibody, there is an interest, possibly a mandate, to screen potential treatment recipients to ensure that they are suitable for treatment. In this review, we summarize progress in the field to date.
\end{abstract}

Key Words Alzheimer's disease · amyloid · imaging · therapeutics $\cdot$ PET-CT

Marwan Sabbagh

marwan.sabbagh@dignityhealh.org

Keshav Anand

keshav.anand@dignityhealth.org

1 St. Joseph's Hospital and Medical Center, 350 W. Thomas Road, Phoenix, AZ 85013, USA

2 Alzhiemer's and Memory Disorders Division, Barrow Neurological Institute, 240 W. Thomas Road, Ste 301, Phoenix, AZ 85013, USA

\section{Introduction}

Alzheimer's disease (AD) is the most common dementia in the world with the neuropathological hallmarks of amyloid plaques $(A \beta)$ and neurofibrillary tangles (composed of tau). The inability to detect these findings during life has led to difficulty in diagnosing cases of dementia without a clear-cut clinical picture.

The prevalence of this disease is increasing, with the number of affected individuals expected to triple by 2050 [1]. The advent of amyloid positive emission tomography (PET) imaging to detect amyloid in living patients is challenging how complicated cases can be diagnosed. Previously, histopathological detection of amyloid plaques required the use of thioflavin-S or Congo red staining postmortem [2]. Now, radioligands can be used in PET imaging with binding to cortical $\mathrm{A} \beta$ allowing detection of in vivo amyloid plaques. As research in the field continues to advance, 3 Food and Drug Administration (FDA)-approved compounds have suggested good safety profiles. Extensive research continues into improving the effectiveness of these agents and the accuracy of scan interpretation.

\section{Appropriate Use Criteria}

The Amyloid Imaging Task Force of the Alzheimer's Association and the Society of Nuclear Medicine and Molecular Imaging are comprised of members who provided appropriate use criteria for scans for particular clinical scenarios. A "dementia expert" with training and/or certification in neurology or psychiatry must determine whether a patient receives an amyloid scan. Using evidence demonstrating the congruence of fluorine-based agents with Pittsburgh Compound B (PIB), the taskforce came up with a set of guidelines for whether scans would be appropriate in a given setting $[3,4]$. 
Situations in which amyloid imaging would be considered appropriate include, for example, when a patient presents with a concern about cognitive performance and $\mathrm{AD}$ is a potential diagnosis; the detection of amyloid via imaging can confirm or deny clinical diagnostic probability and therefore influence future management. Such situations include patients with mild cognitive impairment (MCI) without identifiable cause, ambiguous cases of dementia, or early-onset dementia [3].

Situations in which amyloid imaging would not be considered appropriate include patients with probable $\mathrm{AD}$ given clinical and historical data, quantifying dementia, a sole indication of family history or genetic propensity for $\mathrm{AD}$ without other symptoms, an ambiguous cognitive complaint, asymptomatic patients, or for nonmedical screening [3].

As a result, situations that can alter patient care and therefore directly affect management, are the ones where amyloid imaging is thought to make its mark. Rater training programs exist with certification required for participants to be eligible for reading studies. As a result, such a tool can have a powerful impact on future medical therapy and diagnostic tests. Additionally, the value of knowing the diagnosis for patients allows them to manage their personal and professional goals accordingly [3].

\section{FDA Approval/Centers of Medicare and Medicaid Services Coverage}

To date, 3 compounds have been approved by the FDA for amyloid imaging: ${ }^{18} \mathrm{~F}$-Florbetaben (Neuraceq), ${ }^{18} \mathrm{~F}$-Florbetapir (Amyvid), and ${ }^{18}$ F-Flutemetamol (Vizamyl). The FDA also mandated that readings of amyloid imaging studies be qualified using a binary system of positive or negative scans. While studies have demonstrated the practical use of amyloid imaging in distinguishing $\mathrm{AD}$ from other clinical mimics, there is limited coverage from the Centers of Medicare and Medicaid Services (CMS) with regard to amyloid PET imaging. Currently, CMS will cover 1 amyloid PET for a patient that is enrolled in a specific clinical study conforming to the indications outlined by CMS [5]. Out-of-pocket costs can range upwards of $\$ 3000$. They recommend that these scans should be used as an adjunct when trying to make the diagnosis of $\mathrm{AD}$. While a positive scan may allow one with the right clinical history to make the diagnosis of $\mathrm{AD}$, sole use of this scan should not be used to justify the diagnosis. They recommend that negative scans may predict with high accuracy that a patient does not have AD.

\section{Compounds of Interest}

\section{$\mathrm{N}$-Methyl-[11C]2-(4'-Methylaminophenyl) -6-Hydroxybenzothiazole: PIB}

PIB is related to the amyloid binding dye, Thioflavin-S, and is a benzothiazole with the ability to bind to amyloid in the brain.
The compound was determined to have properties similar to many PET radiotracers in preclinical investigations. The first clinical study of PIB demonstrated the ability of significant retention within the frontal cortex (1.94-fold greater in $\mathrm{AD}$ than in controls) with additional areas of significant retention seen in the parietal, temporal, and occipital lobes. Retention was noted in the striatum in patients with $\mathrm{AD}$, which has been shown in previous studies. Areas often spared by AD did not demonstrate significant retention of PIB. PIB also demonstrated the ability to discriminate between healthy young controls and older patients, with no significant retention seen in controls [6]. Subsequent studies were able to demonstrate congruence of findings, with retention of PIB and diagnosis consistent with $\mathrm{AD}[7-10]$. Equivocal findings in patients with PIB retention and clinical diagnoses of MCI are variable likely owing to cofounders, including amnesia, depression, and alternative dementias playing a role.

A systematic review identifying nine potential studies with 274 participants (112 of whom developed AD) demonstrated disparities in the methodology of scan acquisition (e.g., time to scan from injection of PIB and PIB dosage) along with their interpretations (e.g., standard PIB thresholds, measurements of retention, retention detecting regions, and follow-up). The goal of this review was to investigate the accuracy of PIB scans in predicting conversion from MCI to AD. Inclusion criteria for the systematic review included individuals diagnosed with MCI according to a battery of neuropsychological tests and checklists of activities of daily living (ADL). Excluded patients were those with attributable causes of amnesia such as alcohol, drugs, traumatic brain injury, or other known diagnoses. Additionally, studies varied with respect to average mini-mental state examination (MMSE) scores, and diagnostic criteria for MCI. The sensitivity of these studies ranged from $83 \%$ to $100 \%$, while specificity ranged from $46 \%$ to $88 \%$. The poor specificity suggests that PIB may be a biomarker of other diseases such as vascular causes of MCI or cerebral amyloid angiopathy as PIB binds to $A \beta$ in vessel walls in addition to plaques. Additionally, duration of follow-up was poorly assessed as the trials included in this analysis had a minimum of 1-year follow-up with significant variations in follow-up duration among trials, leading to mixed results given the small number of trials. A fitted receiver-operating characteristic curve was compatible with a positive likelihood ratio of 2.3 and a negative likelihood ratio of 0.07 for conversion to from MCI to AD. A sensitivity analysis to determine whether a preset standard would affect the results demonstrated no change [11]. However, the specificity for predicting accuracy of conversion from $\mathrm{MCI}$ to $\mathrm{AD}$ was improved in patients with longer follow-up periods [12]. These studies demonstrate the value of amyloid imaging to potentially identifying complex cases of AD. While the prospect of PIB in predicting AD has been documented, studies still demonstrate superiority of biochemical and histopathological 
diagnoses [13]. A major limitation of PIB includes its short half-life $(\sim 20 \mathrm{~min})$ with few facilities capable of its production and the necessity of an on-site cyclotron. Novel agents [flourine-18 $\left({ }^{18} \mathrm{~F}\right)$-based compounds] have longer half-lives allowing for use outside the scope of research [14]. Nevertheless, the growing ability of imaging as a viable marker for $\mathrm{AD}$ has allowed for the development of other amyloid imaging agents.

\section{2-(1-\{6-[(2-[ ${ }^{18}$ F]Fluoroethyl)(Methyl)Amino] -2-Naphthyl\}-Ethylidene)Malononitrile}

2-(1-\{6-[(2-[ $\left[{ }^{18} \mathrm{~F}\right]$ fluoroethyl)(methyl)amino $\left.]-2-n a p h t h y l\right\}-$ ethylidene)malononitrile (FDNNP) is a fluorine-based marker, derived from the lipophilic compound di- $N$-nonyl phthalate (DNNP) for imaging AD, and is not an FDA-approved PET imaging ligand. Although this was the first marker to detect amyloid through imaging, it has not gained traction, owing to several limitations. This marker has a unique capability of being able to detect amyloid plaque along with neurofibrillary tangles. It also provides unique detection of AD pathology in the hippocampus $[15,16]$. However, the ability to bind amyloid plaque, neurofibrillary tangles, Lewy bodies, and prions may hinder the utility of FDNNP for routine imaging to distinguish AD from AD mimics [17-20]. This lack of discrimination along with poor signal-to-noise ratio is the rationale for the limited scope of this agent [21]. Additionally, studies have shown that this ligand has weaker affinity for amyloid than PIB does [22].

\section{$\left[{ }^{18} \mathrm{~F}\right] \mathrm{AV}-45$ or Florbetapir}

Florbetapir was developed by Avid Radiopharmaceuticals, which was later acquired by Lilly under the brand name Amyvid. FDA approval for florbetapir came in 2012. The longer half-life and similar selectivity of certain fluorinebased agents for cortical areas similar to the PIB compound allowed for further research into the utility of these agents for AD. Florbetapir is thought to have a binding pattern similar to PIB with a high affinity for $A \beta$ plaques. A study of $14 \operatorname{cog}$ nitively normal individuals and 12 patients with $\mathrm{AD}$ received PET scans with florbetapir and PIB with results demonstrating a group discrimination area under the curve of 0.9 for florbetapir compared versus 1.0 for PIB [23]. A separate study in which 32 patients had undergone a PIB scan followed by a florbetapir study approximately 18 months later allowed for evaluation of cortical retention of these ligands with significant composite cortical binding correlation $(p<0.001)$ [24].

A prospective cohort study of 59 participants and variable cognitive status allowed for evaluation of florbetapir with respect to confirmation of findings at autopsy. Five nuclear medicine physicians were assigned the role of labeling scans as positive or negative for amyloid. Study participants had autopsies within 2 years of scans. Findings demonstrated a sensitivity and specificity of $92 \%$ and $100 \%$, respectively, for detection of moderate-to-frequent plaques if scanned 2 years prior to autopsy, and a sensitivity and specificity of $96 \%$ and $100 \%$, respectively, if scanned 1 year prior to autopsy. This study demonstrates the effectiveness of using an ordinal classification of positive or negative to interpret amyloid imaging at the patient level [25].

A concern for amyloid imaging has been whether the presence of confounding conditions can affect the results of an amyloid scan, for example in patients with $\mathrm{AD}$ and concomitant Lewy bodies. A postmortem study compared 38 individuals with clinically and pathologically defined $\mathrm{AD}$ with 17 cases without $\mathrm{AD}$, with all patients having had prior florbetapir scans. The patients with $\mathrm{AD}$ were further stratified into groups based on concurrent pathology, including Lewy bodies, white matter thinning, cerebral amyloid angiopathy, argyrophilic grains, and TAR DNA-binding protein 43 . Patients with $\mathrm{AD}$ demonstrated increased retention with higher standardized uptake value ratios (SUVR) values compared with patients without AD. The SUVR involves calculating the radioactivity of a specific region compared with another reference range. An interesting side note of this study was that patients with $\mathrm{AD}$ and Lewy bodies had significantly lower SUVRs compared with patients with AD without Lewy bodies [26]. This study demonstrates the ability of florbetapir PET scans to discriminate the presence of amyloid, despite the high clinical/pathologic variability of $\mathrm{AD}$.

Florbetapir scans in patients with normal cognition, MCI, and $\mathrm{AD}$ have demonstrated that patients who were found to be amyloid-positive were predicted and exhibited progressive worsening in cognitive and functional status compared with those who were amyloid-negative. A 36-month follow-up study with 69 cognitively normal, $52 \mathrm{MCI}$, and 31 probable patients with $\mathrm{AD}$ demonstrated the difference between amyloid-positive and amyloid-negative patients with respect to decline in functional and cognitive tests over time. A battery of tests was conducted at baseline and at 36 months, including the Alzheimer's Disease Assessment Scale (ADAS) score, Clinical Dementia Rating Sum of Boxes (CDR-SB), MMSE, ADL, digital symbol substitution (DSS), verbal fluency (animal/vegetable), Wechsler logical memory scale, and Wechsler Memory Scale immediate recall. Those with normal cognition and amyloid-positive scans demonstrated poorer performance on the ADAS-Cognitive Subscale test (ADASCog), DSS, and verbal fluency $(p<0.05)$. Those with MCI and amyloid-positive scans demonstrated inferior performance compared with controls on the ADAS-Cog, DSS, verbal fluency, and the MMSE $(p<0.05)$. Similar patients with $\mathrm{AD}$ demonstrated significantly poorer performance in verbal fluency and the MMSE $(p<0.05)$. All amyloid-positive patients, regardless of cognitive status, demonstrated greater decline in performance on the CDR-SB; however, all groups 
demonstrated no significant decline on the ADL or the Wechsler logical memory scale [27].

With the potential for inter-reader variability in qualitative studies demonstrating differences in reading, adjunctive tools have demonstrated concordance between readers with regard to patients being labeled as amyloid-positive or amyloid-negative. The use of an adjunct for determining SUVRs demonstrated agreement between 5 readers in 27 of 30 scans versus 20 of 30 scans without such a tool $(p=0.06)$ [28]. A semiautomated method for quantifying florbetapir scans with threshold mean cortical SUVR of 1.1 yielded $100 \%$ agreement in cognitively normal patients. Thirty-eight of 39 patients with pathology-proven amyloid had mean cortical SUVRs $>1.1$ [29]. The establishment of a standard can further augment interpretation of florbetapir scans thereby decreasing the chance of ambiguity.

\section{$\left[{ }^{18}\right.$ F $]$ '-F-PiB or Flutemetamol}

Flutemetamol is another ${ }^{18} \mathrm{~F}$-based compound which has demonstrated the ability to bind to $A \beta$. Flutametamol was developed by GE Healthcare, under the brand name Vizamyl. This compound is similar to PIB and hence has structural similarities to Thioflavin-S. Flutemetamol was initially shown in phase I trials to have a good safety profile with affinity to $A \beta$ and increased cortical-to-cerebellar uptake in a small samples size of 8 patients with clinically probable $\mathrm{AD}$ and 8 healthy controls [30]. Phase II trials demonstrated 25 of 27 patients with clinical AD having amyloid-positive scans, and 1 of 15 control patients having a positive scan corresponding to a sensitivity and specificity of $93.1 \%$ and $93.3 \%$, respectively. The study also compared the performance of flutemetamol to PIB with cortical SUVRs for these patients ranging from 0.89 to 0.92 . There was a low degree of test-retest variability, indicating precision of this biomarker [31]. These initial trials led to the favorable promise of flutemetamol given its structural similarity to PIB, reproducibility, and diagnostic capabilities.

Phase III trials aimed at demonstrating the sensitivity and specificity of amyloid PET imaging with flutemetamol using the histopathologic gold standard at autopsy. This study included patients over the age of 55 years with a terminal prognosis of $<1$ year. Sixty-eight patients were autopsied, showing 25 amyloid-negative and 43 amyloid-positive brains via neuropathology. The sensitivity for PET only ranged from $81 \%$ to $93 \%$, while the specificity ranged from $44 \%$ to $92 \%$. Among the 5 readers, 4 of 5 readers had a specificity of $>80 \%$. While the sensitivity and specificity improved with the addition of computerized tomography (CT), these results were not significant. Intrareader concordance was improved with the addition of CT. There was 1 reader who was an outlier in this study. For the majority of the images, there were 6 false-negative cases and 2 false-positive cases. Two of the false-negative cases and 2 of the false-positive cases were patients with dementia with Lewy bodies (DLB). The 2 false-negative cases became positive when CT was used to help with the anatomic details. The remaining 2 false-negative cases were borderline [32]. An additional study aimed to determine the ability of flutemetamol to detect amyloid by PET imaging in different phases of amyloid deposition and found that this approach detects a positive signal in advanced phases of amyloid deposition (phase 4-5). This suggests that PET imaging may be affected by severity of dementia [33].

Further studies aimed to assess similarities between flutemetamol and PIB. A study of 36 patients with AD, 68 patients with $\mathrm{MCI}$, and 62 controls (stratified by age) demonstrated a strong correlation between the 2 compounds $(p<0.001)$, along with the ability to discriminate patients with clinically diagnosed $\mathrm{AD}$ from older healthy controls with a sensitivity and specificity of $97.2 \%$ and $85.3 \%$, respectively [34]. However, another study demonstrated that there is increased concordance between both biomarkers, depending on the region of interest (ROI) for comparison. This study included 32 patients with normal cognition with an attempt to identify preclinical AD. The findings showed that SUVRs with reference regions being the cerebellar gray matter as opposed to the pons resulted in improved concordance of flutemetamol and PIB (Spearman correlation 0.84). Hence, there are different variables which affect the quantitative results of amyloid imaging [35].

With the use of software, studies have implicated an automated technique to classify scans as positive or negative when a threshold for detection is established. A cohort of 68 patients confirmed neuropathologically to have amyloid plaques, along with a test cohort of 33 patients with clinically probable $\mathrm{AD}, 80$ patients with MCI, and 59 healthy controls were used for this study. Additionally, 105 healthy individuals were used to determine a threshold SUVR for the purposes of the experiment. Ranking SUVRs from the autopsy cohort with ROC analysis allowed for evaluation of the automated methodology to best characterize scans by use of thresholds either 2 or 2.5 SDs above the mean. Reference regions included the pons, cerebellar gray matter, or whole cerebellum. Composite regions for analysis were stratified as small, narrow, or large. The results demonstrated that the use of the pons allowed for a larger AUC compared with the cerebellum. The use of a small region for analysis of comparison in the pons with a SUVR threshold of 0.62 allowed for the highest sensitivity and specificity of $91 \%$ and $88 \%$, respectively. There were 3 false-positives using this region, all of which were positive by visual read. There were 4 false-negatives, and 3 of these were negative by visual read. The concordance with visual reads ranged from $97.1 \%$ to $99.4 \%$, depending on the regions evaluated [36].

\section{$\left[{ }^{18} \mathrm{~F}\right]-\mathrm{AV}-1$ or [F-18]-BAY94-9172 or Florbetaben}

Piramal Imaging developed florbetaben under the brand name Neuraceq. This compound is an ${ }^{18} \mathrm{~F}$-labeled polyethylene glycol stilbene derivative, which demonstrated high specificity 
for $\mathrm{A} \beta$ in vitro without binding to tau, frontotemporal lobe dementia tissue, or DLB tissue [37].

An initial study demonstrated the viability of this tracer with high sensitivity for AD along with the similar discriminating capabilities of PIB. This study also demonstrated the ability of diagnosing $\mathrm{AD}$ from other neurodegenerative disorders $(p<0.001)$, including vascular dementia, DLB, and frontotemporal lobar dementia [38]. A phase 0 trial demonstrated the safety and efficacy of this drug. Ten patients with clinically probable $\mathrm{AD}$ and 10 controls were evaluated, with results demonstrating significantly higher SUVRs in the patients with probable AD compared with controls in areas including the frontal, lateral temporal, occipital, parietal, and cingulate cortices $(p<0.01)$. The tracer was also well tolerated [39].

A phase II trial aimed to determine sensitivity and specificity of PET reads in 81 patients with probable AD by MMSE scores of 18 to 26, and 69 controls. Cortical SUVRs were significantly higher in patients with $\mathrm{AD}$, with the posterior cingulate gyrus as the region demonstrating the best area for discrimination. Visual reads yielded a sensitivity and specificity of $80 \%$ and $91 \%$, respectively [40]. Another phase II study was able to detect amyloid in patients with Down syndrome without clinical evidence of dementia, suggesting the utility of imaging in high-risk patients [41].

A phase III trial evaluated the ability of florbetaben to correlate with histopathologic confirmation of disease. The trial involved 216 participants with 74 deceased patients undergoing autopsy. Forty-six of 47 patients with pathologically proven AD were read as PET-positive, while 24 of 27 brains negative for $\mathrm{A} \beta$ were read as PET-negative, corresponding to a sensitivity and specificity of $97.9 \%$ and $88.9 \%$, respectively, at the patient level. Additional aspects of this trial included looking at 6 key tissue scan-matched ROIs; these included the middle frontal gyrus, occipital cortex, hippocampus, anterior cingulate cortex, posterior cingulate cortex/precuneus, and the cerebellar cortex. The overall sensitivities and specificities were $77.4 \%$ and $94.2 \%$, respectively, for the ROIs and matched controls. Higher specificity was seen in the hippocampus at $100 \%$ with lower sensitivity of $57 \%$, while the remainder of the ROIs in which $\mathrm{A} \beta$ plaques occurred more frequently demonstrated a sensitivity ranging from $82 \%$ to $90 \%$, with specificities ranging from $86 \%$ to $95 \%$. This study additionally demonstrated variability in precision of reads across different sites of interest. Another aspect of this study included superimposed magnetic resonance imaging to correlate brain structures with areas of interest on PET scans along with comparison with postmortem analysis [42, 43]. Another study demonstrated the ability of florbetaben to detect amyloid in the prodromal phase of AD, with high concordance between visual readers and SUVR thresholds for PETpositive and PET-negative reads $(\mathrm{k}=0.96)$. Additionally, this study employed the use of hippocampal volume as measured by magnetic resonance imaging and amnesic MCI as measured by a composite episodic memory (EM) Z-score of less than -1.5 to monitor progression of patients with MCI. The results demonstrated that predictive accuracy using either hippocampal volume or EM scores did not improve accuracy in detection. However, EM scores and PET-positive reads did predict progression to $\mathrm{AD}$. Interestingly, over time, the association between positive SUVR and EM scores declined by 2 years, while the association between EM scores and hippocampal volume became stronger. This suggests that accumulation of $\mathrm{A} \beta$ plateaus while hippocampal volume continues to decrease. Therefore, over time, hippocampal atrophy and not accumulation of $\mathrm{A} \beta$ facilitates dementia progression [44].

Recently, a study performed using a retrospective analysis of 22 patients with MCI or dementia compared the accuracy of PIB, fluorodeoxyglucose (FDG)-PET, and ${ }^{18}$ F-florbetaben PET, and demonstrated significant correlation between all 3 biomarkers for AD $(p<0.001)$. With FDG-PET used to discriminate between different dementias, this study adds promise to the value of amyloid-based imaging early in the diagnosis of patients with dementia for AD [45].

Interestingly, a study in patients with multiple sclerosis demonstrated decreased uptake in diseased white matter compared with healthy white matter. While this study only utilized 12 patients, this may serve as a future area of research for studies to find other utilities of amyloid imaging outside the realm of dementia [46].

While the subjectivity of scans raises the concern of inaccurate readings, studies have demonstrated that with appropriate onsite or virtual training, there is a high degree of correlation with pathological diagnosis [47]. However, further abilities to streamline readings with various techniques, for example using partial volume effect correction, can help improve quantitative analysis of PET scans. The effect of such a technique leads to improved discrimination between patients with AD versus healthy controls as interpretation may be affected by brain atrophy [48].

\section{$\left[{ }^{18}\right.$ F]-AZD4694 or NAV4694}

NAV4694 is a novel ${ }^{18} \mathrm{~F}$-labeled compound undergoing active phase II and III clinical trials through Navidea Pharmaceuticals; the trials are not presently recruiting. Initial in vitro studies demonstrated the high affinity of this compound for $\mathrm{A} \beta$ with less binding in the white matter [49]. Further, clinical trials demonstrated the ability of NAV4694 to discriminate between clinically diagnosed patients with AD and normal control subjects with a high degree of test-retest concordance using the reference Logan approach [50]. As a standard for comparison, a study with 25 controls, 10 patients with MCI, 7 with probable $\mathrm{AD}$, and 3 with probable frontotemporal dementia underwent scans with PIB and NAV4694, with similar regions of interests identified on both scans. The results demonstrated similarity in the cortical SUVRs with the advantage of low white matter 
binding. There was significant correlation between PIB and NAV4694 for cortical SUVR $(p<0.0001)$. This ability to discriminate binding in white matter could prove advantageous in future studies. The current phase II trial aims to predict progression to $\mathrm{AD}$ in patients with $\mathrm{MCI}$, while the phase III trial aims to evaluate efficacy and safety when compared with autopsy [51,52].

\section{Additional Biomarkers}

2-(p-Methylaminophenyl)-7-(2-[ $\left[{ }^{18} \mathrm{~F}\right]$ fluoroethoxy)imidazo[2,1$b$ benzothiazole (FIBT) is another ${ }^{18} \mathrm{~F}$-based compound with preclinical trials in transgenic mice with $A P P / P S 1$ genes matched to controls. Comparison of PET imaging in this model with FIBT, florbetaben, and PIB demonstrated the ability of FIBT to selectively bind cortical amyloid selectively with SUVRs similar to that of PIB [53].

Another ${ }^{18} \mathrm{~F}$-based biomarker, (E)-5-(2-(6- $(2-[18 \mathrm{~F}]-$ fluoroethoxy)-benzo[d]thiazol-2-yl)vinyl)-N,Ndimethylpyridin-2-amine $\left({ }^{18} \mathrm{~F}-7 \mathrm{~b}\right)$ has undergone preclinical trials with promising results. High affinity of ${ }^{18} \mathrm{~F}-7 \mathrm{~b}$ for cortical $\mathrm{A} \beta$ plaques in $\mathrm{AD}$ brain homogenates has been shown with good penetration to enable micro-PET/CT imaging. Preliminary imaging showed higher retention in transgenic AD mice than in wild-type controls demonstrating an additional potential agent for amyloid imaging [54].

\section{Conclusion}

While the prognosis of patients with $\mathrm{AD}$ is still poor, new techniques in the form of amyloid imaging have had some success in identifying patients with AD. Although still relatively new, the success of the ${ }^{18} \mathrm{~F}$-based compounds compared with PIB and neuropathologic correlation provide an avenue through which difficult cases have another opportunity to clarify diagnosis. Identifying cases of amyloid positivity in patients with MCI may be essential towards delineating future disease and prognosis of these patients. Additionally, the utility of such molecular imaging in patients with increased risk of disease whether due to age, positive family history, or genetic propensity still requires further investigation. As a result, the International Working Group has revised the diagnostic criteria, with the International Working Group-2 criteria for $\mathrm{AD}$, allowing that positive biomarkers through amyloid positivity on ${ }^{18} \mathrm{~F}$ imaging or cerebrospinal fluid biomarkers, along with the clinical diagnosis of $\mathrm{AD}$ and its less common subtypes or high-risk presymptomatic patients, dictate the confirmation of a diagnosis and therefore allow for subsequent management [55]. The detection of amyloid can therefore be instrumental in guiding patient management in complex cases.

The Imaging Dementia Evidence for Amyloid Scanning (IDEAS) Study which occurs in collaboration between the Alzheimer's Association, American College of Radiology and the American College of Radiology Imaging Network aims to follow $>18,000$ Medicare patients to determine the value and utility of amyloid imaging. The patients eligible for this study based on the aforementioned appropriate use criteria will be evaluated with aims including the impact of amyloid imaging on patient management along with the impact of imaging on patient morbidity, including, but not limited to, hospital admissions and emergency room visits [56].

While there are no current studies comparing florbetapir, flutemetamol, and florbetaben against one another, this is an area of potential investigation in the future, along with their comparison with PIB as a control to demonstrate the relative similarities and differences between the compounds. This strategy may help delineate advantages with the compounds and also to see how differences in technique affect results.

The positive results of research in the field of amyloid imaging leaves us with questions regarding what to do with clinically diagnosed patients with $\mathrm{AD}$ who have negative scans. Further research in the field will better allow us to determine the probability of having $\mathrm{AD}$ with the clinical symptoms of dementia. Future studies including logistic regression and functional analyses can allow us to better grasp this concept and therefore guide management in these ambiguous cases. An ideal scenario would include a predictive model combining clinical diagnosis and amyloid imaging positivity, which could increase the predictive value of AD. Similar concepts have been incorporated in creating models in the setting of apolipoprotein E-positive and FDG-PETpositive cases [57, 58].

More important than trying to understand the clinical utility of amyloid imaging is recognizing that it might become an important screening tool for determining the utilization of antiamyloid treatments. There are many monoclonal antibodies in development (solanezumab, gantenerumab, crenezumab, aducanumab). Future utilization of the biologics, if approved, will require amyloid imaging for screening purposes to ensure amyloid positivity. The alternative will be cerebrospinal fluid testing as a screening measure.

Additionally, the use of software with consensus threshold values for SUVR along with region of comparison is still being investigated, with future studies likely determining a standardized way to analyze the data. While original studies measured SUVRs, the FDA mandated that binary reads are the standard for practice. As a result, variations in SUVRs can drastically affect results between positive or negative. With further research, we may establish an automated or objective way to interpret scans allowing for minimizing difference in inter-reader variability.

Acknowledgments This work was supported by NIA P30 AG019610 and the Barrow Neurological Institute.

Required Author Forms Disclosure forms provided by the authors are available with the online version of this article. 


\section{References}

1. Querfurth, H. W., \& LaFerla, F. M. (2010). Alzheimer's disease. $N$ Engl J Med 362, 329-344.

2. Serrano-Pozo, A., Frosch, M. P., Masliah, E., \& Hyman, B. T. (2011). Neuropathological Alterations in Alzheimer Disease. Cold Spring Harb Perspect Med 1:a006189.

3. Johnson, K. A., Minoshima, S., Bohnen, N. I., et al. (2013). Appropriate use criteria for amyloid PET: a report of the Amyloid Imaging Task Force, the Society of Nuclear Medicine and Molecular Imaging, and the Alzheimer's Association. Alzheimers Dement 9, e-1-16.

4. Johnson, K. A., Minoshima, S., Bohnen, N. I., et al. (2013). Update on appropriate use criteria for amyloid PET imaging: dementia experts, mild cognitive impairment, and education. $J$ Nucl Med 54, 1011-1013.

5. Jacques, L, Jensen, T., Rollins, J., et al. (2013, September 27). Decision Memo for Beta Amyloid Positron Emission Tomography in Dementia and Neurodegenerative Disease (CAG00431N). Centers for Medicare and Medicaid Services. Available at: https://www.cms.gov/medicare-coverage-database/details/ncadecision-memo.aspx?NCAId=265. Accessed August 5, 2016.

6. Klunk, W. E., Engler, H., Nordberg, A., et al. (2004). Imaging brain amyloid in Alzheimer's disease with Pittsburgh Compound-B. Ann Neurol 55, 306-319.

7. Archer, H. A., Edison, P., Brooks, D. J., et al. (2006). Amyloid load and cerebral atrophy in Alzheimer's disease: an 11C-PIB positron emission tomography study. Ann Neurol 60(1), 145-147.

8. Edison, P., Archer, H. A., Hinz, R., et al. (2007). Amyloid, hypometabolism, and cognition in Alzheimer disease: an [11C]PIB and [18F]FDG PET study. Neurology 68, 501-508.

9. Grimmer, T., Henriksen, G., Wester, H.-J., et al. (2009). Clinical severity of Alzheimer's disease is associated with PIB uptake in PET. Neurobiol Aging, 30, 1902-1909.

10. Rowe, C. C., Ng, S., Ackermann, U., et al. L. (2007). Imaging betaamyloid burden in aging and dementia. Neurology 68, 1718-1725.

11. Zhang, S., Smailagic, N., Hyde, C., et al. (2014). (11)C-PIB-PET for the early diagnosis of Alzheimer's disease dementia and other dementias in people with mild cognitive impairment (MCI). Cochrane Database Syst Rev 7, CD010386.

12. Ma, Y., Zhang, S., Li, J., et al. (2014). Predictive accuracy of amyloid imaging for progression from mild cognitive impairment to Alzheimer disease with different lengths of follow-up: a meta-analysis. [Corrected]. Medicine 93, e150.

13. Niedowicz, D. M., Beckett, T. L., Matveev, S., et al. (2012). Pittsburgh compound $\mathrm{b}$ and the postmortem diagnosis of Alzheimer's disease. Ann Neurol 72, 564-570.

14. Klunk, W. E., \& Mathis, C. A. (2008). The future of amyloid-beta imaging: a tale of radionuclides and tracer proliferation. Curr Opin Neurol 21, 683-687.

15. Shin, J., Kepe, V., Barrio, J. R., \& Small, G. W. (2011). The merits of FDDNP-PET imaging in Alzheimer's disease. J Alzheimers Dis 26(Suppl. 3), 135-145.

16. Small, G. W., Kepe, V., Ercoli, L. M., et al. (2006). PET of brain amyloid and tau in mild cognitive impairment. $N$ Engl $\mathrm{J} \mathrm{Med} \mathrm{355,}$ 2652-2663.

17. Adlard, P. A., Tran, B. A., Finkelstein, D. I., et al. (2014). A review of $\beta$-amyloid neuroimaging in Alzheimer's disease. Front Neurosci $8: 327$.

18. Agdeppa, E. D., Kepe, V., Liu, J., et al. (2001). Binding characteristics of radiofluorinated 6-dialkylamino-2-naphthylethylidene derivatives as positron emission tomography imaging probes for betaamyloid plaques in Alzheimer's disease. J Neurosci 21, RC189.

19. Bresjanac, M., Smid, L. M., Vovko, T. D., Petric, A., Barrio, J. R., \& Popovic, M. (2003). Molecular-imaging probe 2-(1-[6-[(2- fluoroethyl)(methyl) amino]-2-naphthyl]ethylidene) malononitrile labels prion plaques in vitro. $J$ Neurosci 23, 8029-8033.

20. Smid, L. M., Vovko, T. D., Popovic, M., et al. (2006). The 2,6disubstituted naphthalene derivative FDDNP labeling reliably predicts Congo red birefringence of protein deposits in brain sections of selected human neurodegenerative diseases. Brain Pathol 16, 124-130.

21. Shimojo, M., Higuchi, M., Suhara, T., \& Sahara, N. (2015). Imaging multimodalities for dissecting Alzheimer's disease: advanced technologies of positron emission tomography and fluorescence imaging. Front Neurosci 9:482.

22. Thompson, P. W., Ye, L., Morgenstern, J. L., et al. (2009). Interaction of the amyloid imaging tracer FDDNP with hallmark Alzheimer's disease pathologies. J Neurochem 109, 623-630.

23. Wolk, D. A., Zhang, Z., Boudhar, S., Clark, C. M., Pontecorvo, M. J., \& Arnold, S. E. (2012). Amyloid imaging in Alzheimer's disease: comparison of florbetapir and Pittsburgh compound-B positron emission tomography. J Neurol Neurosurg Psychiatry 83, 923 926.

24. Landau, S. M., Breault, C., Joshi, A. D., et al. (2013). Amyloid- $\beta$ imaging with Pittsburgh compound B and florbetapir: comparing radiotracers and quantification methods. J Nucl Med 54(1), 70-77.

25. Clark, C. M., Pontecorvo, M. J., Beach, T. G., et al. (2012). Cerebral PET with florbetapir compared with neuropathology at autopsy for detection of neuritic amyloid- $\beta$ plaques: a prospective cohort study. Lancet Neurol 11, 669-678.

26. Dugger, B. N., Clark, C. M., Serrano, G., et al. (2014). Neuropathologic heterogeneity does not impair florbetapirpositron emission tomography postmortem correlates. $J$ Neuropathol Exp Neurol 73, 72-80.

27. Doraiswamy, P. M., Sperling, R. A., Johnson, K., et al. (2014). Florbetapir F 18 amyloid PET and 36-month cognitive decline: a prospective multicenter study. Mol Psychiatry 19, 1044-1051.

28. Nayate, A. P., Dubroff, J. G., Schmitt, J. E., et al. (2015). Use of standardized uptake value ratios decreases interreader variability of [18F] florbetapir PET brain scan interpretation. AJNR Am $J$ Neuroradiol 36, 1237-1244.

29. Joshi, A. D., Pontecorvo, M. J., Lu, M., Skovronsky, D. M., Mintun, M. A., \& Devous, M. D. (2015). A semiautomated method for quantification of F 18 florbetapir PET images. J Nucl Med 56, 1736-1741.

30. Nelissen, N., Van Laere, K., Thurfjell, L., et al. (2009). Phase 1 study of the Pittsburgh compound B derivative 18F-flutemetamol in healthy volunteers and patients with probable Alzheimer disease. J Nucl Med 50, 1251-1259.

31. Vandenberghe, R., Van Laere, K., Ivanoiu, A., et al. (2010). 18Fflutemetamol amyloid imaging in Alzheimer disease and mild cognitive impairment: a phase 2 trial. Ann Neurol 68, 319-329.

32. Curtis, C., Gamez, J. E., Singh, U., et al. (2015). Phase 3 trial of flutemetamol labeled with radioactive fluorine 18 imaging and neuritic plaque density. JAMA Neurol 72, 287-294.

33. Thal, D. R., Beach, T. G., Zanette, M., et al. (2015). [(18)F]flutemetamol amyloid positron emission tomography in preclinical and symptomatic Alzheimer's disease: specific detection of advanced phases of amyloid- $\beta$ pathology. Alzheimers Dement 11, 975-985.

34. Hatashita, S., Yamasaki, H., Suzuki, Y., Tanaka, K., Wakebe, D., \& Hayakawa, H. (2014). [18F]Flutemetamol amyloid-beta PET imaging compared with [11C]PIB across the spectrum of Alzheimer's disease. Eur J Nucl Med Mol Imaging 41, 290-300.

35. Adamczuk, K., Schaeverbeke, J., Nelissen, N., et al. (2016). Amyloid imaging in cognitively normal older adults: comparison between (18)F-flutemetamol and (11)C-Pittsburgh compound B. Eur J Nucl Med Mol Imaging 43, 142-151.

36. Thurfjell, L., Lilja, J., Lundqvist, R., et al. (2014). Automated quantification of $18 \mathrm{~F}$-flutemetamol PET activity for categorizing scans 
as negative or positive for brain amyloid: concordance with visual image reads. $J$ Nucl Med 55(10), 1623-1628.

37. Fodero-Tavoletti, M. T., Brockschnieder, D., Villemagne, V. L., et al. (2012). In vitro characterization of [18F]-florbetaben, an $A \beta$ imaging radiotracer. $\mathrm{Nucl}$ Med Biol 39, 1042-1048.

38. Villemagne, V. L., Ong, K., Mulligan, R. S., et al. (2011). Amyloid imaging with (18)F-florbetaben in Alzheimer disease and other dementias. $J$ Nucl Med 52(8), 1210-1217.

39. Barthel, H., Luthardt, J., Becker, G., et al. (2011). Individualized quantification of brain $\beta$-amyloid burden: results of a proof of mechanism phase 0 florbetaben PET trial in patients with Alzheimer's disease and healthy controls. Eur J Nucl Med Mol Imaging 38, 1702-1714.

40. Barthel, H., Gertz, H.-J., Dresel, S., et al. (2011). Cerebral amyloid$\beta$ PET with florbetaben (18F) in patients with Alzheimer's disease and healthy controls: a multicentre phase 2 diagnostic study. Lancet Neurol 10, 424-435.

41. Jennings, D., Seibyl, J., Sabbagh, M., et al. (2015). Age dependence of brain $\beta$-amyloid deposition in Down syndrome: An [18F]florbetaben PET study. Neurology 84, 500-507.

42. Sabri, O., Sabbagh, M. N., Seibyl, J., et al. (2015). Florbetaben PET imaging to detect amyloid plaques in Alzheimer disease: Phase 3 study. Alzheimers Dement 11:964-974.

43. Sabri, O., Seibyl, J., Rowe, C., \& Barthel, H. (2015). Beta-amyloid imaging with florbetaben. Clin Transl Imaging, 3, 13-26.

44. Ong, K. T., Villemagne, V. L., Bahar-Fuchs, A., et al. (2015). A $\beta$ imaging with $18 \mathrm{~F}$-florbetaben in prodromal Alzheimer's disease: a prospective outcome study. J Neurol Neurosurg Psychiatry 86, 431-436.

45. Tiepolt, S., Hesse, S., Patt, M., et al. (2016). Early [(18)F]florbetaben and [(11)C]PiB PET images are a surrogate biomarker of neuronal injury in Alzheimer's disease. Eur J Nucl Med Mol Imaging 43, 1700-1709.

46. Matías-Guiu, J. A., Cabrera-Martín, M. N., Matías-Guiu, J., et al. (2015). Amyloid PET imaging in multiple sclerosis: an (18)Fflorbetaben study. BMC Neurol 15, 243.

47. Seibyl, J., Catafau, A. M., Barthel, H., et al. (2016). Impact of training method on the robustness of the visual assessment of 18F-florbetaben PET scans: results from a Phase 3 trial. $J$ Nucl Med 57:900-906.
48. Rullmann, M., Dukart, J., Hoffmann, K.-T et al. (2016). Partialvolume effect correction improves quantitative analysis of $18 \mathrm{f}-$ florbetaben $\beta$-amyloid PET scans. J Nucl Med 57, 198-203.

49. Juréus, A., Swahn, B.-M., Sandell, J., et al. (2010). Characterization of AZD4694, a novel fluorinated Abeta plaque neuroimaging PET radioligand. J Neurochem 114, 784-794.

50. Cselényi, Z., Jönhagen, M. E., Forsberg, A., et al. (2012). Clinical validation of $18 \mathrm{~F}$-AZD4694, an amyloid- $\beta$-specific PET radioligand. $J$ Nucl Med 53(3), 415-424.

51. Navidea Biopharmaceuticals. (2013). Beta-amyloid imaging with [18F]NAV4694 positron emission tomography (PET) in predicting progression to Alzheimer's disease (AD) in subjects with mild cognitive impairment (MCI) (NAV4-04). Available at: https://clinicaltrials.gov/ct2/show/NCT01812213. Accessed August 5, 2016.

52. Navidea Biopharmaceuticals. (2015). A phase 3 clinical trial to evaluate the efficacy and safety of [18F]NAV4694 PET for detection of cerebral beta-amyloid when compared with postmortem histopathology. Available at: https://clinicaltrials.gov/ct2/show/NCT01886820. Accessed August 5, 2016.

53. Yousefi, B. H., von Reutern, B., Scherübl, D., et al. (2015). FIBT versus florbetaben and $\mathrm{PiB}$ : a preclinical comparison study with amyloid-PET in transgenic mice. EJNMMI Res 5:20.

54. Sundaram, G. S., Dhavale, D., Prior, J. L., et al. (2015). Synthesis, characterization, and preclinical validation of a PET radiopharmaceutical for interrogating $\mathrm{A} \beta$ ( $\beta$-amyloid) plaques in Alzheimer's disease. EJNMMI Res 5, 112.

55. Dubois, B., Feldman, H. H., Jacova, C., et al. (2014). Advancing research diagnostic criteria for Alzheimer's disease: the IWG-2 criteria. Lancet Neurol 13, 614-629.

56. Imaging Dementia-Evidence for Amyloid Scanning. (n.d.). Available at: http://www.ideas-study.org/about/. Accessed August 5, 2016.

57. Relkin, N. R., Kwon, Y. J., Tsai, J., \& Gandy, S. (1996). The National Institute on Aging/Alzheimer's Association recommendations on the application of apolipoprotein E genotyping to Alzheimer's disease. Ann N Y Acad Sci 802, 149-176.

58. Silverman, D., Torosyan, N., Manne, M., Durcanova, B., Dahlbom, M., \& Apostolova, L. (2014). Value of amyloid imaging for predicting conversion to dementia in MCI subjects with initially indeterminate FDG-PET scans. Alzheimers Dement 10, P18-P19. 